\title{
Dirac System with Discontinuities at Two Points
}

\author{
Fatma Hıra and Nihat Altınışık \\ Department of Mathematics, Science and Arts Faculty, The University of Ondokuz Mayis, 55139 Samsun, Turkey
}

Correspondence should be addressed to Fatma Hira; fatma.hira@omu.edu.tr

Received 9 September 2013; Revised 30 January 2014; Accepted 11 February 2014; Published 17 March 2014

Academic Editor: Alexander Domoshnitsky

Copyright (C) 2014 F. Hıra and N. Altınışık. This is an open access article distributed under the Creative Commons Attribution License, which permits unrestricted use, distribution, and reproduction in any medium, provided the original work is properly cited.

We deal with a regular Dirac system which has discontinuities at two points and contains eigenparameter in a boundary condition and two transmission conditions. We investigate asymptotic behaviour of eigenvalues and corresponding eigenfunctions of this Dirac system and construct Green's function.

\section{Introduction}

We consider the Dirac system

$$
\begin{gathered}
u_{2}^{\prime}(x)-p_{1}(x) u_{1}(x)=\lambda u_{1}(x), \\
u_{1}^{\prime}(x)+p_{2}(x) u_{2}(x)=-\lambda u_{2}(x), \quad x \in I, \\
B_{1}(u):=\beta_{1} u_{1}(a)+\beta_{2} u_{2}(a)=0, \\
B_{2}(u):=\left(\lambda \alpha_{1}^{\prime}-\alpha_{1}\right) u_{1}(b)-\left(\lambda \alpha_{2}^{\prime}-\alpha_{2}\right) u_{2}(b)=0, \\
T_{1}(u):=u_{1}\left(c_{1}^{-}\right)-\delta u_{1}\left(c_{1}^{+}\right)=0, \\
T_{2}(u):=u_{2}\left(c_{1}^{-}\right)-\delta u_{2}\left(c_{1}^{+}\right)+\lambda u_{1}\left(c_{1}^{-}\right)=0, \\
T_{3}(u):=\delta u_{1}\left(c_{2}^{-}\right)-\gamma u_{1}\left(c_{2}^{+}\right)=0, \\
T_{4}(u):=\delta u_{2}\left(c_{2}^{-}\right)-\gamma u_{2}\left(c_{2}^{+}\right)+\lambda u_{1}\left(c_{2}^{-}\right)=0,
\end{gathered}
$$

where $I=\left[a, c_{1}\right) \cup\left(c_{1}, c_{2}\right) \cup\left(c_{2}, b\right], a<c_{1}<c_{2}<$ $b ; \lambda \in \mathbb{C}$; the real valued functions $p_{1}(\cdot)$ and $p_{2}(\cdot)$ are continuous in $\left[a, c_{1}\right),\left(c_{1}, c_{2}\right)$ and $\left(c_{2}, b\right]$ and have finite limits $p_{1}\left(c_{1}^{ \pm}\right):=\lim _{x \rightarrow c_{1}^{ \pm}} p_{1}(x), p_{1}\left(c_{2}^{ \pm}\right):=\lim _{x \rightarrow c_{2}^{ \pm}} p_{1}(x), p_{2}\left(c_{1}^{ \pm}\right):=$ $\lim _{x \rightarrow c_{1}^{ \pm}} p_{2}(x), p_{2}\left(c_{2}^{ \pm}\right):=\lim _{x \rightarrow c_{2}^{ \pm}} p_{2}(x) ; \alpha_{i}, \alpha_{i}^{\prime}, \beta_{i} \in \mathbb{R}(i=$ $1,2), \beta_{1}^{2}+\beta_{2}^{2} \neq 0 ; \delta, \gamma \in \mathbb{R}, \delta>0, \gamma \neq 0 ; c_{i}^{-}:=c_{i}-0, c_{i}^{+}:=$ $c_{i}+0(i=1,2)$ and $\rho:=\left(\alpha_{1}^{\prime} \alpha_{2}-\alpha_{1} \alpha_{2}^{\prime}\right)>0$.

Boundary-value problems with transmission (or discontinuity) conditions inside the interval often appear in mathematical physics, mechanics, electronics, geophysics, and other branches of natural sciences (see $[1,2])$. References [3-5] are examples of works with boundary conditions depending linearly on an eigenparameter and transmission conditions at the point of discontinuity for Sturm Liouville problem.

The basic and comprehensive results about Dirac operators were given in [6]. The oscillation property and asymptotic formulas for the eigenvalues of Dirac systems were given in [7] and the derivative sampling theorem to compute eigenvalues of Dirac systems was used in $[8,9]$. Continuous Dirac systems have been investigated in these works. Works in direction of Dirac systems with an internal point of discontinuity are few (see [10-13]). Sampling theories associated with discontinuous Dirac systems were investigated in [11, 12] (also the problem in [12] contains eigenparameter in a boundary condition). Direct and inverse problems for Dirac operators with discontinuity inside an interval were studied in [13]. Dirac operators with eigenparameter dependent on both boundary conditions and one of the discontinuity conditions were investigated in [10]. In these discontinuous works, Dirac systems had only one point of discontinuity.

We examine Dirac system which has two points of discontinuity and contains at the same time an eigenparameter in a boundary condition and two transmission conditions in this paper. Dirac system which has two points of discontinuity does not exist as far as we know. Our investigation will be a good example for discontinuous Dirac systems. Firstly, we give some spectral properties of eigenvalues and eigenfunctions and then obtain asymptotic formulas 
for eigenvalues and corresponding eigenfunctions. Finally, we construct Green's function of the problem (1)-(7).

\section{Spectral Properties}

To formulate a theoretic approach to problem (1)-(7), let $L:=$ $L_{2}\left(a, c_{1}\right) \oplus L_{2}\left(c_{1}, c_{2}\right) \oplus L_{2}\left(c_{2}, b\right)$ and $\mathbb{C}^{3}:=\mathbb{C} \oplus \mathbb{C} \oplus \mathbb{C}$ and we define the Hilbert space $H=L \oplus \mathbb{C}^{3}$ with an inner product

$$
\begin{aligned}
\langle U(\cdot), V(\cdot)\rangle_{H} \\
:=\int_{a}^{c_{1}} \mathbf{u}^{\top}(x) \overline{\mathbf{v}}(x) d x+\delta^{2} \int_{\mathcal{c}_{1}}^{c_{2}} \mathbf{u}^{\top}(x) \overline{\mathbf{v}}(x) d x \\
\quad+\gamma^{2} \int_{\mathcal{C}_{2}}^{b} \mathbf{u}^{\top}(x) \overline{\mathbf{v}}(x) d x+\frac{\gamma^{2}}{\rho} h_{1} \overline{k_{1}}+h_{2} \overline{k_{2}}+\delta h_{3} \overline{k_{3}}
\end{aligned}
$$

where $T$ denotes the matrix transpose,

$$
\begin{gathered}
U(x)=\left(\begin{array}{c}
\mathbf{u}(x) \\
h_{1} \\
h_{2} \\
h_{3}
\end{array}\right), \quad V(x)=\left(\begin{array}{c}
\mathbf{v}(x) \\
k_{1} \\
k_{2} \\
k_{3}
\end{array}\right) \in H, \\
\mathbf{u}(x)=\left(\begin{array}{c}
u_{1}(x) \\
u_{2}(x)
\end{array}\right), \quad \mathbf{v}(x)=\left(\begin{array}{c}
v_{1}(x) \\
v_{2}(x)
\end{array}\right) \in L, \\
u_{i}(\cdot), v_{i}(\cdot) \in L_{2}(a, b) \quad(i=1,2), \\
h_{j}, k_{j} \in \mathbb{C} \quad(j=1,2,3) .
\end{gathered}
$$

For functions $u(x)$, which are defined on $I$ and have finite limit $u\left(c_{1}^{ \pm}\right):=\lim _{x \rightarrow c_{1}^{ \pm}} u(x)$ and $u\left(c_{2}^{ \pm}\right):=\lim _{x \rightarrow c_{2}^{ \pm}} u(x)$, by $u_{(1)}(x), u_{(2)}(x)$, and $u_{(3)}(x)$, we denote the functions

$$
\begin{aligned}
& u_{(1)}(x)= \begin{cases}u(x), & x \in\left[a, c_{1}\right), \\
u\left(c_{1}^{-}\right), & x=c_{1},\end{cases} \\
& u_{(2)}(x)= \begin{cases}u\left(c_{1}^{+}\right), & x=c_{1}, \\
u(x), & x \in\left(c_{1}, c_{2}\right), \\
u\left(c_{2}^{-}\right), & x=c_{2},\end{cases} \\
& u_{(3)}(x)= \begin{cases}u(x), & x \in\left(c_{2}, b\right], \\
u\left(c_{2}^{+}\right), & x=c_{2},\end{cases}
\end{aligned}
$$

which are defined on $I_{1}:=\left[a, c_{1}\right], I_{2}:=\left[c_{1}, c_{2}\right]$, and $I_{3}:=$ $\left[c_{2}, b\right]$, respectively.

Lemma 1. The eigenvalues of the problem (1)-(7) are real.

Proof. Assume the contrary that $\lambda_{0}$ is a nonreal eigenvalue of problem (1)-(7). Let $\left(\begin{array}{l}u_{1}(x) \\ u_{2}(x)\end{array}\right)$ be a corresponding (nontrivial) eigenfunction. By (1), we have

$$
\begin{aligned}
& \frac{d}{d x}\left\{u_{1}(x) \bar{u}_{2}(x)-\bar{u}_{1}(x) u_{2}(x)\right\} \\
& \quad=\left(\bar{\lambda}_{0}-\lambda_{0}\right)\left\{\left|u_{1}(x)\right|^{2}+\left|u_{2}(x)\right|^{2}\right\}, \quad x \in I .
\end{aligned}
$$

Integrating the above equation through $\left[a, c_{1}\right],\left[c_{1}, c_{2}\right]$, and $\left[c_{2}, b\right]$, we obtain

$$
\begin{gathered}
\left(\bar{\lambda}_{0}-\lambda_{0}\right)\left(\int_{a}^{c_{1}}\left(\left|u_{1}(x)\right|^{2}+\left|u_{2}(x)\right|^{2}\right) d x\right) \\
=u_{1}\left(c_{1}^{-}\right) \bar{u}_{2}\left(c_{1}^{-}\right)-\bar{u}_{1}\left(c_{1}^{-}\right) u_{2}\left(c_{1}^{-}\right) \\
-\left(u_{1}(a) \bar{u}_{2}(a)-\bar{u}_{1}(a) u_{2}(a)\right), \\
\left(\bar{\lambda}_{0}-\lambda_{0}\right)\left(\int_{c_{1}}^{c_{2}}\left(\left|u_{1}(x)\right|^{2}+\left|u_{2}(x)\right|^{2}\right) d x\right) \\
=u_{1}\left(c_{2}^{-}\right) \bar{u}_{2}\left(c_{2}^{-}\right)-\bar{u}_{1}\left(c_{2}^{-}\right) u_{2}\left(c_{2}^{-}\right) \\
-\left(u_{1}\left(c_{1}^{+}\right) \bar{u}_{2}\left(c_{1}^{+}\right)-\bar{u}_{1}\left(c_{1}^{+}\right) u_{2}\left(c_{1}^{+}\right)\right),
\end{gathered}
$$

$$
\begin{aligned}
\left(\bar{\lambda}_{0}-\lambda_{0}\right)\left(\int_{\mathcal{c}_{2}}^{b}\left(\left|u_{1}(x)\right|^{2}+\left|u_{2}(x)\right|^{2}\right) d x\right) \\
=u_{1}(b) \bar{u}_{2}(b)-\bar{u}_{1}(b) u_{2}(b) \\
-\left(u_{1}\left(c_{2}^{+}\right) \bar{u}_{2}\left(c_{2}^{+}\right)-\bar{u}_{1}\left(c_{2}^{+}\right) u_{2}\left(c_{2}^{+}\right)\right) .
\end{aligned}
$$

Then from boundary conditions (2)-(3) and transmission conditions (4)-(7), we have, respectively,

$$
u_{1}(a) \bar{u}_{2}(a)-\bar{u}_{1}(a) u_{2}(a)=0
$$

$$
\begin{gathered}
u_{1}(b) \bar{u}_{2}(b)-\bar{u}_{1}(b) u_{2}(b)=-\frac{\rho\left(\bar{\lambda}_{0}-\lambda_{0}\right)\left|u_{2}(b)\right|^{2}}{\left|\lambda_{0} \alpha_{1}^{\prime}-\alpha_{1}\right|^{2}}, \\
u_{1}\left(c_{1}^{-}\right) \bar{u}_{2}\left(c_{1}^{-}\right)-\bar{u}_{1}\left(c_{1}^{-}\right) u_{2}\left(c_{1}^{-}\right) \\
=\delta^{2}\left(u_{1}\left(c_{1}^{+}\right) \bar{u}_{2}\left(c_{1}^{+}\right)-\bar{u}_{1}\left(c_{1}^{+}\right) u_{2}\left(c_{1}^{+}\right)\right), \\
\delta^{2}\left(u_{1}\left(c_{2}^{-}\right) \bar{u}_{2}\left(c_{2}^{-}\right)-\bar{u}_{1}\left(c_{2}^{-}\right) u_{2}\left(c_{2}^{-}\right)\right) \\
=\gamma^{2}\left(u_{1}\left(c_{2}^{+}\right) \bar{u}_{2}\left(c_{2}^{+}\right)-\bar{u}_{1}\left(c_{2}^{+}\right) u_{2}\left(c_{2}^{+}\right)\right) .
\end{gathered}
$$

Since $\bar{\lambda}_{0} \neq \lambda_{0}$, it follows from (13)-(16) and (12) that

$$
\begin{aligned}
& \int_{a}^{c_{1}}\left(\left|u_{1}(x)\right|^{2}+\left|u_{2}(x)\right|^{2}\right) d x+\delta^{2} \int_{c_{1}}^{c_{2}}\left(\left|u_{1}(x)\right|^{2}+\left|u_{2}(x)\right|^{2}\right) d x \\
& +\gamma^{2} \int_{\mathcal{C}_{2}}^{b}\left(\left|u_{1}(x)\right|^{2}+\left|u_{2}(x)\right|^{2}\right) d x=-\frac{\rho \gamma^{2}\left|u_{2}(b)\right|^{2}}{\left|\lambda_{0} \alpha_{1}^{\prime}-\alpha_{1}\right|^{2}} .
\end{aligned}
$$

This contradicts the conditions $\int_{a}^{c_{1}}\left(\left|u_{1}(x)\right|^{2}+\left|u_{2}(x)\right|^{2}\right) d x+$ $\delta^{2} \int_{c_{1}}^{c_{2}}\left(\left|u_{1}(x)\right|^{2}+\left|u_{2}(x)\right|^{2}\right) d x+\gamma^{2} \int_{\mathcal{C}_{2}}^{b}\left(\left|u_{1}(x)\right|^{2}+\left|u_{2}(x)\right|^{2}\right) d x>0$ and $\rho>0$. Consequently, $\lambda_{0}$ must be real. 
Lemma 2. Let $\lambda$ and $\mu$ be two different eigenvalues of problem (1)-(7). Then the corresponding eigenfunctions $\mathbf{u}(x, \lambda)$ and $\mathbf{v}(x, \mu)$ of this problem satisfy the following equality:

$$
\begin{aligned}
& \int_{a}^{c_{1}} \mathbf{u}^{\top}(x, \lambda) \mathbf{v}(x, \mu) d x+\delta^{2} \int_{c_{1}}^{c_{2}} \mathbf{u}^{\top}(x, \lambda) \mathbf{v}(x, \mu) d x \\
& +\gamma^{2} \int_{\mathcal{C}_{2}}^{b} \mathbf{u}^{\top}(x, \lambda) \mathbf{v}(x, \mu) d x+\frac{\gamma^{2}}{\rho} h_{1} k_{1}+h_{2} k_{2}+\delta h_{3} k_{3}=0 .
\end{aligned}
$$

One must note that, since the eigenfunctions are real valued, the left hand side in the last expression coincides with the inner product in $H$.

Now one will construct a special fundamental system of solutions of (1). By virtue of Theorem 1.1 in [14], one will define two solutions of (1),

$$
\phi(\cdot, \lambda)=\left(\begin{array}{l}
\phi_{1}(\cdot, \lambda) \\
\phi_{2}(\cdot, \lambda)
\end{array}\right), \quad \chi(\cdot, \lambda)=\left(\begin{array}{l}
\chi_{1}(\cdot, \lambda) \\
\chi_{2}(\cdot, \lambda)
\end{array}\right)
$$

where

$$
\begin{aligned}
& \phi_{1}(x, \lambda)= \begin{cases}\phi_{11}(x, \lambda), & x \in\left[a, c_{1}\right), \\
\phi_{12}(x, \lambda), & x \in\left(c_{1}, c_{2}\right), \\
\phi_{13}(x, \lambda), & x \in\left(c_{2}, b\right],\end{cases} \\
& \phi_{2}(x, \lambda)= \begin{cases}\phi_{21}(x, \lambda), & x \in\left[a, c_{1}\right), \\
\phi_{22}(x, \lambda), & x \in\left(c_{1}, c_{2}\right), \\
\phi_{23}(x, \lambda), & x \in\left(c_{2}, b\right],\end{cases} \\
& \chi_{1}(x, \lambda)= \begin{cases}\chi_{11}(x, \lambda), & x \in\left[a, c_{1}\right), \\
\chi_{12}(x, \lambda), & x \in\left(c_{1}, c_{2}\right), \\
\chi_{13}(x, \lambda), & x \in\left(c_{2}, b\right],\end{cases} \\
& \chi_{2}(x, \lambda)= \begin{cases}\chi_{21}(x, \lambda), & x \in\left[a, c_{1}\right), \\
\chi_{22}(x, \lambda), & x \in\left(c_{1}, c_{2}\right), \\
\chi_{23}(x, \lambda), & x \in\left(c_{2}, b\right],\end{cases}
\end{aligned}
$$

as follows. Let one consider the next initial value problem:

$$
\begin{gathered}
u_{2}^{\prime}(x)-p_{1}(x) u_{1}(x)=\lambda u_{1}(x), \\
u_{1}^{\prime}(x)+p_{2}(x) u_{2}(x)=-\lambda u_{2}(x), \quad x \in\left(a, c_{1}\right) \\
u_{1}(a)=\beta_{2}, \quad u_{2}(a)=-\beta_{1} .
\end{gathered}
$$

By virtue of Theorem 1.1 in [14], this problem has a unique solution $\mathbf{u}=\left(\begin{array}{c}\phi_{11}(x, \lambda) \\ \phi_{21}(x, \lambda)\end{array}\right)$, which is an entire function of $\lambda \epsilon$ $\mathbb{C}$ for each fixed $x \in\left[a, c_{1}\right]$. Similarly, employing the same method as in the proof of Theorem 1.1 in [14], one sees that the problem

$$
\begin{aligned}
& u_{2}^{\prime}(x)-p_{1}(x) u_{1}(x)=\lambda u_{1}(x), \\
& u_{1}^{\prime}(x)+p_{2}(x) u_{2}(x)=-\lambda u_{2}(x), \quad x \in\left(c_{2}, b\right) \\
& u_{1}(b)=\lambda \alpha_{2}^{\prime}-\alpha_{2}, \quad u_{2}(b)=\lambda \alpha_{1}^{\prime}-\alpha_{1},
\end{aligned}
$$

has a unique solution $\mathbf{u}=\left(\begin{array}{l}\chi_{13}(x, \lambda) \\ \chi_{23}(x, \lambda)\end{array}\right)$, which is an entire function of parameter $\lambda \in \mathbb{C}$ for each fixed $x \in\left[c_{2}, b\right]$.

Now the functions $\phi_{1 i}(x, \lambda)$ and $\phi_{2 i}(x, \lambda)$ are defined in terms of $\phi_{1 j}(x, \lambda)$ and $\phi_{2 j}(x, \lambda), i=2,3, j=1,2$, respectively, as follows. The initial value problem

$$
\begin{gathered}
u_{2}^{\prime}(x)-p_{1}(x) u_{1}(x)=\lambda u_{1}(x), \\
u_{1}^{\prime}(x)+p_{2}(x) u_{2}(x)=-\lambda u_{2}(x), \quad x \in\left(c_{1}, c_{2}\right), \\
u_{1}\left(c_{1}\right)=\frac{1}{\delta} \phi_{11}\left(c_{1}^{-}, \lambda\right), \\
u_{2}\left(c_{1}\right)=\frac{1}{\delta}\left\{\phi_{21}\left(c_{1}^{-}, \lambda\right)+\lambda \phi_{11}\left(c_{1}^{-}, \lambda\right)\right\}
\end{gathered}
$$

has a unique solution $\mathbf{u}=\left(\begin{array}{l}\phi_{12}(x, \lambda) \\ \phi_{22}(x, \lambda)\end{array}\right)$ for each $\lambda \in \mathbb{C}$, and the initial value problem

$$
\begin{aligned}
& u_{2}^{\prime}(x)-p_{1}(x) u_{1}(x)=\lambda u_{1}(x), \\
& u_{1}^{\prime}(x)+p_{2}(x) u_{2}(x)=-\lambda u_{2}(x), \quad x \in\left(c_{2}, b\right), \\
& u_{1}\left(c_{2}\right)=\frac{\delta}{\gamma} \phi_{12}\left(c_{2}^{-}, \lambda\right) \\
& u_{2}\left(c_{2}\right)=\frac{1}{\gamma}\left\{\delta \phi_{22}\left(c_{2}^{-}, \lambda\right)+\lambda \phi_{12}\left(c_{2}^{-}, \lambda\right)\right\},
\end{aligned}
$$

has a unique solution $\mathbf{u}=\left(\begin{array}{c}\phi_{13}(x, \lambda) \\ \phi_{23}(x, \lambda)\end{array}\right)$ for each $\lambda \in \mathbb{C}$.

Similarly, the functions $\chi_{1 j}(x, \lambda)$ and $\chi_{2 j}(x, \lambda)$ are defined in terms of $\chi_{1 i}(x, \lambda)$ and $\chi_{2 i}(x, \lambda), i=2,3, j=1,2$, respectively, as follows. The initial value problem

$$
\begin{gathered}
u_{2}^{\prime}(x)-p_{1}(x) u_{1}(x)=\lambda u_{1}(x), \\
u_{1}^{\prime}(x)+p_{2}(x) u_{2}(x)=-\lambda u_{2}(x), \quad x \in\left(c_{1}, c_{2}\right), \\
u_{1}\left(c_{2}\right)=\frac{\gamma}{\delta} \chi_{13}\left(c_{2}^{+}, \lambda\right), \\
u_{2}\left(c_{2}\right)=\frac{\gamma}{\delta}\left\{\chi_{23}\left(c_{2}^{+}, \lambda\right)-\frac{\lambda}{\delta} \chi_{13}\left(c_{2}^{+}, \lambda\right)\right\}
\end{gathered}
$$

has a unique solution $\mathbf{u}=\left(\begin{array}{l}\chi_{12}(x, \lambda) \\ \chi_{22}(x, \lambda)\end{array}\right)$ for each $\lambda \in \mathbb{C}$, and the initial value problem

$$
\begin{gathered}
u_{2}^{\prime}(x)-p_{1}(x) u_{1}(x)=\lambda u_{1}(x), \\
u_{1}^{\prime}(x)+p_{2}(x) u_{2}(x)=-\lambda u_{2}(x), \quad x \in\left(a, c_{1}\right), \\
u_{1}\left(c_{1}\right)=\delta \chi_{12}\left(c_{1}^{+}, \lambda\right) \\
u_{2}\left(c_{1}\right)=\delta\left\{\chi_{22}\left(c_{1}^{+}, \lambda\right)-\lambda \chi_{12}\left(c_{1}^{+}, \lambda\right)\right\}
\end{gathered}
$$

has a unique solution $\mathbf{u}=\left(\begin{array}{l}\chi_{11}(x, \lambda) \\ \chi_{21}(x, \lambda)\end{array}\right)$ for each $\lambda \in \mathbb{C}$.

Hence, $\phi(\cdot, \lambda)$ satisfies (1) on $I$, boundary condition (2), and transmission conditions $(4)-(7)$ and $\chi(\cdot, \lambda)$ satisfies $(1)$ on $I$, boundary condition (3), and transmission conditions (4) $-(7)$. 
Let $W(\boldsymbol{\phi}, \boldsymbol{\chi})(\cdot, \lambda)$ denote the Wronskians of $\phi(\cdot, \lambda)$ and $\chi(\cdot, \lambda)$ defined by

$$
W(\phi, \chi)(\cdot, \lambda):=\left|\begin{array}{ll}
\phi_{1}(\cdot, \lambda) & \phi_{2}(\cdot, \lambda) \\
\chi_{1}(\cdot, \lambda) & \chi_{2}(\cdot, \lambda)
\end{array}\right|
$$

It is obvious that the Wronskians

$$
\begin{aligned}
\omega_{i}(\lambda) & :=W(\phi, \chi)(\cdot, \lambda) \\
& =\phi_{1 i}(x, \lambda) \chi_{2 i}(x, \lambda)-\phi_{2 i}(x, \lambda) \chi_{1 i}(x, \lambda), \quad x \in I_{i}
\end{aligned}
$$

are independent of $x \in I_{i}(i=1,2,3)$ and are entire functions. Taking into account (26), (28), (30), and (32), a short calculation gives

$$
\omega_{1}(\lambda)=\delta^{2} \omega_{2}(\lambda)=\gamma^{2} \omega_{3}(\lambda)
$$

for each $\lambda \in \mathbb{C}$.

Corollary 3. The zeros of the functions $\omega_{1}(\lambda), \omega_{2}(\lambda)$, and $\omega_{3}(\lambda)$ coincide.

Then, one may take into consideration the characteristic function $\omega(\lambda)$ as

$$
\omega(\lambda):=\omega_{1}(\lambda)=\delta^{2} \omega_{2}(\lambda)=\gamma^{2} \omega_{3}(\lambda)
$$

Lemma 4. All eigenvalues of problem (1)-(7) are just zeros of the function $\omega(\lambda)$. Moreover, every zero of $\omega(\lambda)$ has multiplicity one.

Proof. Since the functions $\phi_{1}(x, \lambda)$ and $\phi_{2}(x, \lambda)$ satisfy the boundary condition (2) and transmission conditions (4)-(7), to find the eigenvalues of problem (1)-(7), we have to insert the functions $\phi_{1}(x, \lambda)$ and $\phi_{2}(x, \lambda)$ in the boundary condition (3) and find the roots of this equation.

By (1), we obtain, for $\lambda, \mu \in \mathbb{C}, \lambda \neq \mu$,

$$
\begin{aligned}
& \frac{d}{d x}\left\{\phi_{1}(x, \lambda) \phi_{2}(x, \mu)-\phi_{1}(x, \mu) \phi_{2}(x, \lambda)\right\} \\
& \quad=(\mu-\lambda)\left\{\phi_{1}(x, \lambda) \phi_{1}(x, \mu)+\phi_{2}(x, \lambda) \phi_{2}(x, \mu)\right\} .
\end{aligned}
$$

Integrating the above equation through $\left[a, c_{1}\right],\left[c_{1}, c_{2}\right]$, and $\left[c_{2}, b\right]$ and taking into account the initial conditions (23), (26), (28), (30), and (32), we obtain

$$
\begin{gathered}
(\lambda-\mu)\left\{\delta^{2} \phi_{12}\left(c_{1}^{+}, \mu\right) \phi_{12}\left(c_{1}^{+}, \lambda\right)+\gamma^{2} \phi_{13}\left(c_{2}^{+}, \lambda\right) \phi_{13}\left(c_{2}^{+}, \mu\right)\right\} \\
+\gamma^{2}\left(\phi_{13}(b, \lambda) \phi_{23}(b, \mu)-\phi_{13}(b, \mu) \phi_{23}(b, \lambda)\right) \\
=(\mu-\lambda)\left\{\int _ { a } ^ { c _ { 1 } } \left(\phi_{11}(x, \lambda) \phi_{11}(x, \mu)\right.\right. \\
\left.+\phi_{21}(x, \lambda) \phi_{21}(x, \mu)\right) d x \\
+\delta^{2} \int_{c_{1}}^{c_{2}}\left(\phi_{12}(x, \lambda) \phi_{12}(x, \mu)\right. \\
\left.+\phi_{22}(x, \lambda) \phi_{22}(x, \mu)\right) d x \\
+\gamma^{2} \int_{\mathcal{C}_{2}}^{b}\left(\phi_{13}(x, \lambda) \phi_{13}(x, \mu)\right. \\
\left.\left.+\phi_{23}(x, \lambda) \phi_{23}(x, \mu)\right) d x\right\} .
\end{gathered}
$$

Dividing both sides of (38) by $(\lambda-\mu)$ and letting $\mu \rightarrow \lambda$, we reach the relation

$$
\begin{aligned}
& \delta^{2}\left(\phi_{12}\left(c_{1}^{+}, \lambda\right)\right)^{2}+\gamma^{2}\left(\phi_{13}\left(c_{2}^{+}, \lambda\right)\right)^{2} \\
&+ \gamma^{2}\left(\phi_{23}(b, \lambda) \frac{\partial \phi_{13}(b, \lambda)}{\partial \lambda}-\phi_{13}(b, \lambda) \frac{\partial \phi_{23}(b, \lambda)}{\partial \lambda}\right) \\
&=-\left\{\int_{a}^{c_{1}}\left(\left|\phi_{11}(x, \lambda)\right|^{2}+\left|\phi_{21}(x, \lambda)\right|^{2}\right) d x\right. \\
&+ \delta^{2} \int_{\mathcal{c}_{1}}^{c_{2}}\left(\left|\phi_{12}(x, \lambda)\right|^{2}+\left|\phi_{22}(x, \lambda)\right|^{2}\right) d x \\
&+\left.\gamma^{2} \int_{\mathcal{C}_{2}}^{b}\left(\left|\phi_{13}(x, \lambda)\right|^{2}+\left|\phi_{23}(x, \lambda)\right|^{2}\right) d x\right\} .
\end{aligned}
$$

We show that equation

$\omega(\lambda)=\gamma^{2}\left(\left(\lambda \alpha_{1}^{\prime}-\alpha_{1}\right) \phi_{13}(b, \lambda)-\left(\lambda \alpha_{2}^{\prime}-\alpha_{2}\right) \phi_{23}(b, \lambda)\right)=0$

has only simple roots. Assume the converse; that is, (40) has a double root $\lambda_{0}$. Then the following two equations hold:

$$
\begin{gathered}
\left(\lambda_{0} \alpha_{1}^{\prime}-\alpha_{1}\right) \phi_{13}\left(b, \lambda_{0}\right)-\left(\lambda_{0} \alpha_{2}^{\prime}-\alpha_{2}\right) \phi_{23}\left(b, \lambda_{0}\right)=0 \\
\alpha_{1}^{\prime} \phi_{13}\left(b, \lambda_{0}\right)+\left(\lambda_{0} \alpha_{1}^{\prime}-\alpha_{1}\right) \frac{\partial \phi_{13}\left(b, \lambda_{0}\right)}{\partial \lambda}-\alpha_{2}^{\prime} \phi_{23}\left(b, \lambda_{0}\right) \\
-\left(\lambda_{0} \alpha_{2}^{\prime}-\alpha_{2}\right) \frac{\partial \phi_{23}\left(b, \lambda_{0}\right)}{\partial \lambda}=0 .
\end{gathered}
$$


Since $\rho \neq 0$ and $\lambda_{0}$ is real, then $\left(\lambda_{0} \alpha_{1}^{\prime}-\alpha_{1}\right)^{2}+$ $\left(\lambda_{0} \alpha_{2}^{\prime}-\alpha_{2}\right)^{2} \neq 0$, from (41),

$$
\begin{gathered}
\phi_{13}\left(b, \lambda_{0}\right)=\frac{\left(\lambda_{0} \alpha_{2}^{\prime}-\alpha_{2}\right)}{\left(\lambda_{0} \alpha_{1}^{\prime}-\alpha_{1}\right)} \phi_{23}\left(b, \lambda_{0}\right), \\
\frac{\partial \phi_{13}\left(b, \lambda_{0}\right)}{\partial \lambda}=\frac{\rho \phi_{23}\left(b, \lambda_{0}\right)}{\left(\lambda_{0} \alpha_{1}^{\prime}-\alpha_{1}\right)^{2}}+\frac{\left(\lambda_{0} \alpha_{2}^{\prime}-\alpha_{2}\right)}{\left(\lambda_{0} \alpha_{1}^{\prime}-\alpha_{1}\right)} \frac{\partial \phi_{23}\left(b, \lambda_{0}\right)}{\partial \lambda} .
\end{gathered}
$$

Combining (42) and (39), with $\lambda=\lambda_{0}$, we obtain

$$
\begin{gathered}
\delta^{2}\left(\phi_{12}\left(c_{1}^{+}, \lambda\right)\right)^{2}+\gamma^{2}\left(\phi_{13}\left(c_{2}^{+}, \lambda\right)\right)^{2}+\frac{\rho \gamma^{2}\left(\phi_{23}\left(b, \lambda_{0}\right)\right)^{2}}{\left(\lambda_{0} \alpha_{1}^{\prime}-\alpha_{1}\right)^{2}} \\
=-\left\{\int_{a}^{c_{1}}\left(\left|\phi_{11}(x, \lambda)\right|^{2}+\left|\phi_{21}(x, \lambda)\right|^{2}\right) d x\right. \\
+\delta^{2} \int_{c_{1}}^{c_{2}}\left(\left|\phi_{12}(x, \lambda)\right|^{2}+\left|\phi_{22}(x, \lambda)\right|^{2}\right) d x \\
+\gamma^{2} \int_{\mathcal{C}_{2}}^{b}\left(\phi_{13}(x, \lambda) \phi_{13}(x, \mu)\right. \\
\left.\left.+\phi_{23}(x, \lambda) \phi_{23}(x, \mu)\right) d x\right\}
\end{gathered}
$$

contradicting the assumption $\rho>0$.

\section{Asymptotic Approximate Formulas}

Now using variation of constants (see [14]), we will transform (1), (20), (23), (26), and (28) into the integral equations

$$
\begin{aligned}
\phi_{11}(x, \lambda)= & \beta_{2} \cos (\lambda(x-a))+\beta_{1} \sin (\lambda(x-a)) \\
& -\int_{a}^{x} \sin (\lambda(x-y)) p_{1}(y) \phi_{11}(y, \lambda) d y \\
& -\int_{a}^{x} \cos (\lambda(x-y)) p_{2}(y) \phi_{21}(y, \lambda) d y,
\end{aligned}
$$

$$
\phi_{21}(x, \lambda)=\beta_{2} \sin (\lambda(x-a))-\beta_{1} \cos (\lambda(x-a))
$$$$
\begin{aligned}
& +\int_{a}^{x} \cos (\lambda(x-y)) p_{1}(y) \phi_{11}(y, \lambda) d y \\
& -\int_{a}^{x} \sin (\lambda(x-y)) p_{2}(y) \phi_{21}(y, \lambda) d y,
\end{aligned}
$$

$$
\begin{aligned}
\phi_{12}(x, \lambda)= & \frac{1}{\delta} \phi_{11}\left(c_{1}^{-}, \lambda\right) \cos \left(\lambda\left(x-c_{1}\right)\right) \\
& -\frac{1}{\delta}\left(\phi_{21}\left(c_{1}^{-}, \lambda\right)+\lambda \phi_{11}\left(c_{1}^{-}, \lambda\right)\right) \sin \left(\lambda\left(x-c_{1}\right)\right) \\
& -\int_{\mathcal{c}_{1}}^{x} \sin (\lambda(x-y)) p_{1}(y) \phi_{12}(y, \lambda) d y \\
& -\int_{c_{1}}^{x} \cos (\lambda(x-y)) p_{2}(y) \phi_{22}(y, \lambda) d y,
\end{aligned}
$$

$$
\begin{aligned}
\phi_{22}(x, \lambda)= & \frac{1}{\delta} \phi_{11}\left(c_{1}^{-}, \lambda\right) \sin \left(\lambda\left(x-c_{1}\right)\right) \\
& +\frac{1}{\delta}\left(\phi_{21}\left(c_{1}^{-}, \lambda\right)+\lambda \phi_{11}\left(c_{1}^{-}, \lambda\right)\right) \cos \left(\lambda\left(x-c_{1}\right)\right) \\
& +\int_{\mathcal{c}_{1}}^{x} \cos (\lambda(x-y)) p_{1}(y) \phi_{12}(y, \lambda) d y \\
& -\int_{\mathcal{c}_{1}}^{x} \sin (\lambda(x-y)) p_{2}(y) \phi_{22}(y, \lambda) d y
\end{aligned}
$$

$$
\begin{aligned}
\phi_{13}(x, \lambda)= & \frac{\delta}{\gamma} \phi_{12}\left(c_{2}^{-}, \lambda\right) \cos \left(\lambda\left(x-c_{2}\right)\right) \\
& -\frac{1}{\gamma}\left(\delta \phi_{22}\left(c_{2}^{-}, \lambda\right)+\lambda \phi_{12}\left(c_{2}^{-}, \lambda\right)\right) \sin \left(\lambda\left(x-c_{2}\right)\right) \\
& -\int_{\mathcal{C}_{2}}^{x} \sin (\lambda(x-y)) p_{1}(y) \phi_{13}(y, \lambda) d y \\
& -\int_{\mathcal{C}_{2}}^{x} \cos (\lambda(x-y)) p_{2}(y) \phi_{23}(y, \lambda) d y, \\
\phi_{23}(x, \lambda)= & \frac{\delta}{\gamma} \phi_{12}\left(c_{2}^{-}, \lambda\right) \sin \left(\lambda\left(x-c_{2}\right)\right) \\
& +\frac{1}{\gamma}\left(\delta \phi_{22}\left(c_{2}^{-}, \lambda\right)+\lambda \phi_{12}\left(c_{2}^{-}, \lambda\right)\right) \cos \left(\lambda\left(x-c_{2}\right)\right) \\
& +\int_{\mathcal{C}_{2}}^{x} \cos (\lambda(x-y)) p_{1}(y) \phi_{13}(y, \lambda) d y \\
& -\int_{\mathcal{C}_{2}}^{x} \sin (\lambda(x-y)) p_{2}(y) \phi_{23}(y, \lambda) d y .
\end{aligned}
$$

For $|\lambda| \rightarrow \infty$, the solutions $\phi_{1 i}(x, \lambda)$ and $\phi_{2 i}(x, \lambda)(i=$ $1,2,3)$ have the following asymptotic representation uniformly with respect to $x, x \in I$, cf. [14]:

$$
\begin{aligned}
\phi_{11}(x, \lambda)= & {\left[\beta_{2} \cos (\lambda(x-a))+\beta_{1} \sin (\lambda(x-a))\right] } \\
& +O\left(\frac{1}{\lambda} e^{\tau(x-a)}\right), \\
\phi_{21}(x, \lambda)= & {\left[\beta_{2} \sin (\lambda(x-a))-\beta_{1} \cos (\lambda(x-a))\right] } \\
& +O\left(\frac{1}{\lambda} e^{\tau(x-a)}\right), \\
\phi_{12}(x, \lambda)= & \frac{-\lambda}{\delta}\left[\beta_{2} \cos \left(\lambda\left(c_{1}-a\right)\right)+\beta_{1} \sin \left(\lambda\left(c_{1}-a\right)\right)\right] \\
& \times \sin \left(\lambda\left(x-c_{1}\right)\right)+O\left(e^{\tau(x-a)}\right), \\
\phi_{22}(x, \lambda)= & \frac{\lambda}{\delta}\left[\beta_{2} \cos \left(\lambda\left(c_{1}-a\right)\right)+\beta_{1} \sin \left(\lambda\left(c_{1}-a\right)\right)\right] \\
& \times \cos \left(\lambda\left(x-c_{1}\right)\right)+O\left(e^{\tau(x-a)}\right),
\end{aligned}
$$




$$
\begin{aligned}
\phi_{13}(x, \lambda)= & \frac{\lambda^{2}}{\delta \gamma}\left[\beta_{2} \cos \left(\lambda\left(c_{1}-a\right)\right)+\beta_{1} \sin \left(\lambda\left(c_{1}-a\right)\right)\right] \\
& \times \sin \left(\lambda\left(c_{2}-c_{1}\right)\right) \sin \left(\lambda\left(x-c_{2}\right)\right) \\
& +O\left(\lambda e^{\tau(x-a)}\right), \\
\phi_{23}(x, \lambda)= & -\frac{\lambda^{2}}{\delta \gamma}\left[\beta_{2} \cos \left(\lambda\left(c_{1}-a\right)\right)+\beta_{1} \sin \left(\lambda\left(c_{1}-a\right)\right)\right] \\
& \times \sin \left(\lambda\left(c_{2}-c_{1}\right)\right) \cos \left(\lambda\left(x-c_{2}\right)\right) \\
& +O\left(\lambda e^{\tau(x-a)}\right),
\end{aligned}
$$

where $\tau=|\operatorname{Im} \lambda|$.

By substituting the obtained asymptotic formulas for $\phi_{1 i}(x, \lambda)$ and $\phi_{2 i}(x, \lambda)(i=1,2,3)$ in the definitions of $\omega(\lambda)$, we can establish the following theorem.

Theorem 5. Let $\tau=|\operatorname{Im} \lambda|$. Then the characteristic function $\omega(\lambda)$ has the following asymptotic representation:

$$
\begin{aligned}
\omega(\lambda)=\frac{\lambda^{3} \gamma}{\delta}\{ & \alpha_{1}^{\prime}\left[\beta_{2} \cos \left(\lambda\left(c_{1}-a\right)\right)+\beta_{1} \sin \left(\lambda\left(c_{1}-a\right)\right)\right] \\
& \times \sin \left(\lambda\left(c_{2}-c_{1}\right)\right) \sin \left(\lambda\left(b-c_{2}\right)\right) \\
& +\alpha_{2}^{\prime}\left[\beta_{2} \cos \left(\lambda\left(c_{1}-a\right)\right)+\beta_{1} \sin \left(\lambda\left(c_{1}-a\right)\right)\right] \\
& \left.\times \sin \left(\lambda\left(c_{2}-c_{1}\right)\right) \cos \left(\lambda\left(b-c_{2}\right)\right)\right\} \\
+ & O\left(\lambda^{2} e^{\tau(b-a)}\right) .
\end{aligned}
$$

By putting $\sin z=\left(e^{i z}-e^{-i z}\right) / 2 i$ and $\cos z=\left(e^{i z}+e^{-i z}\right) / 2$ in (46), one derives that

$$
\omega(\lambda)=\widetilde{\omega}(\lambda)+O\left(\lambda^{2} e^{\tau(b-a)}\right),
$$

where

$$
\begin{gathered}
\widetilde{\omega}(\lambda) \\
=\frac{\lambda^{3} \gamma}{\delta}\left\{e^{2 \lambda i(b-a)}\left(-\frac{\alpha_{1}^{\prime} \beta_{2}}{8}-\frac{\alpha_{1}^{\prime} \beta_{1}}{8 i}+\frac{\alpha_{2}^{\prime} \beta_{2}}{8 i}-\frac{\alpha_{2}^{\prime} \beta_{1}}{8}\right)\right. \\
+e^{2 \lambda i\left(b-a+c_{1}-c_{2}\right)}\left(\frac{\alpha_{1}^{\prime} \beta_{2}}{8}+\frac{\alpha_{1}^{\prime} \beta_{1}}{8 i}-\frac{\alpha_{2}^{\prime} \beta_{2}}{8 i}+\frac{\alpha_{2}^{\prime} \beta_{1}}{8}\right) \\
+e^{2 \lambda i\left(c_{2}-a\right)}\left(\frac{\alpha_{1}^{\prime} \beta_{2}}{8}+\frac{\alpha_{1}^{\prime} \beta_{1}}{8 i}+\frac{\alpha_{2}^{\prime} \beta_{2}}{8 i}-\frac{\alpha_{2}^{\prime} \beta_{1}}{8}\right) \\
+e^{2 \lambda i\left(c_{1}-a\right)}\left(-\frac{\alpha_{1}^{\prime} \beta_{2}}{8}-\frac{\alpha_{1}^{\prime} \beta_{1}}{8 i}-\frac{\alpha_{2}^{\prime} \beta_{2}}{8 i}+\frac{\alpha_{2}^{\prime} \beta_{1}}{8}\right)
\end{gathered}
$$

$$
\begin{aligned}
& +e^{2 \lambda i\left(b-c_{1}\right)}\left(-\frac{\alpha_{1}^{\prime} \beta_{2}}{8}+\frac{\alpha_{1}^{\prime} \beta_{1}}{8 i}+\frac{\alpha_{2}^{\prime} \beta_{2}}{8 i}+\frac{\alpha_{2}^{\prime} \beta_{1}}{8}\right) \\
& +e^{2 \lambda i\left(c_{2}-c_{1}\right)}\left(\frac{\alpha_{1}^{\prime} \beta_{2}}{8}-\frac{\alpha_{1}^{\prime} \beta_{1}}{8 i}+\frac{\alpha_{2}^{\prime} \beta_{2}}{8 i}+\frac{\alpha_{2}^{\prime} \beta_{1}}{8}\right) \\
& +e^{2 \lambda i\left(b-c_{2}\right)}\left(\frac{\alpha_{1}^{\prime} \beta_{2}}{8}-\frac{\alpha_{1}^{\prime} \beta_{1}}{8 i}-\frac{\alpha_{2}^{\prime} \beta_{2}}{8 i}-\frac{\alpha_{2}^{\prime} \beta_{1}}{8}\right) \\
& \left.+\left(-\frac{\alpha_{1}^{\prime} \beta_{2}}{8}+\frac{\alpha_{1}^{\prime} \beta_{1}}{8 i}-\frac{\alpha_{2}^{\prime} \beta_{2}}{8 i}-\frac{\alpha_{2}^{\prime} \beta_{1}}{8}\right)\right\} .
\end{aligned}
$$

Lemma 6. Let $\left\{\alpha_{i}\right\}_{i=1}^{p}$ be the set of real numbers satisfying the inequalities $\alpha_{0}>\alpha_{1}>\cdots>\alpha_{p-1}>0$ and $\left\{\beta_{i}\right\}_{i=1}^{p}$ the set of complex numbers. If $\beta_{p} \neq 0$, then the roots of the equation

$$
e^{\alpha_{0} \lambda}+\beta_{1} e^{\alpha_{1} \lambda}+\cdots+\beta_{p-1} e^{\alpha_{p-1} \lambda}+\beta_{p}=0
$$

have the form

$$
\lambda_{n}=\frac{2 n \pi i}{\alpha_{0}}+\Psi(n) \quad(n=0, \pm 1, \ldots)
$$

where $\Psi(n)$ is a bounded sequence [15].

Theorem 7. The eigenvalues $\lambda_{n}$ of problem (1)-(7) have the following asymptotic formula:

$$
\lambda_{n}=\frac{n \pi}{(b-a)}+O(1)
$$

Proof. By using Lemma 6, $\widetilde{\omega}(\lambda)=0$ has an infinite number of roots $\widetilde{\lambda}_{n}$ with asymptotic expression

$$
\tilde{\lambda}_{n}=\frac{n \pi}{(b-a)}+\Psi(n), \quad \sup _{n}|\Psi(n)|<\infty
$$

where $\Psi(n)=O(1 / n)$. By applying well-known Rouche's theorem, which assets that if $f(s)$ and $g(s)$ are analytic inside and on a closed contour $\Gamma$, and $|g(s)|<|f(s)|$ on $\Gamma$, then $f(s)$ and $f(s)+g(s)$ have the same number of zeros inside $\Gamma$, and then we obtain

$$
\begin{gathered}
\lambda_{n}=\tilde{\lambda}_{n}+O(1), \\
\lambda_{n}=\frac{n \pi}{(b-a)}+O(1) .
\end{gathered}
$$

By putting (51) in the (45), we obtain the following asymptotic formulae of the eigen-vector-functions $\phi\left(\cdot, \lambda_{n}\right)$ : 


$$
\phi\left(x, \lambda_{n}\right)= \begin{cases}\left(\begin{array}{l}
\left(\beta_{2} \cos \left(\lambda_{n}(x-a)\right)+\beta_{1} \sin \left(\lambda_{n}(x-a)\right)\right)+O\left(\frac{1}{n}\right) \\
\left(\beta_{2} \sin \left(\lambda_{n}(x-a)\right)-\beta_{1} \cos \left(\lambda_{n}(x-a)\right)\right)+O\left(\frac{1}{n}\right)
\end{array}\right), & x \in\left[a, c_{1}\right), \\
\left(\begin{array}{l}
-\frac{\lambda_{n}}{\delta}\left(\beta_{2} \cos \left(\lambda_{n}\left(c_{1}-a\right)\right)+\beta_{1} \sin \left(\lambda_{n}\left(c_{1}-a\right)\right)\right) \sin \left(\lambda_{n}\left(x-c_{1}\right)\right)+O(1) \\
\frac{\lambda_{n}}{\delta}\left(\beta_{2} \cos \left(\lambda_{n}\left(c_{1}-a\right)\right)+\beta_{1} \sin \left(\lambda_{n}\left(c_{1}-a\right)\right)\right) \cos \left(\lambda_{n}\left(x-c_{1}\right)\right)+O(1)
\end{array}\right), & x \in\left(c_{1}, c_{2}\right), \\
\left(\begin{array}{l}
\frac{\lambda_{n}^{2}}{\delta \gamma}\left(\beta_{2} \cos \left(\lambda_{n}\left(c_{1}-a\right)\right)+\beta_{1} \sin \left(\lambda_{n}\left(c_{1}-a\right)\right)\right) \sin \left(\lambda_{n}\left(c_{2}-c_{1}\right)\right) \sin \left(\lambda_{n}\left(x-c_{2}\right)\right)+O(n) \\
-\frac{\lambda_{n}^{2}}{\delta \gamma}\left(\beta_{2} \cos \left(\lambda_{n}\left(c_{1}-a\right)\right)+\beta_{1} \sin \left(\lambda_{n}\left(c_{1}-a\right)\right)\right) \sin \left(\lambda_{n}\left(c_{2}-c_{1}\right)\right) \cos \left(\lambda_{n}\left(x-c_{2}\right)\right)+O(n)
\end{array}\right), & x \in\left(c_{2}, b\right],\end{cases}
$$

where

$$
\phi\left(x, \lambda_{n}\right)= \begin{cases}\left(\begin{array}{l}
\phi_{11}\left(x, \lambda_{n}\right) \\
\phi_{21}\left(x, \lambda_{n}\right)
\end{array}\right), & x \in\left[a, c_{1}\right), \\
\left(\begin{array}{l}
\phi_{12}\left(x, \lambda_{n}\right) \\
\phi_{22}\left(x, \lambda_{n}\right)
\end{array}\right), & x \in\left(c_{1}, c_{2}\right), \\
\left(\begin{array}{l}
\phi_{13}\left(x, \lambda_{n}\right) \\
\phi_{23}\left(x, \lambda_{n}\right)
\end{array}\right), & x \in\left(c_{2}, b\right] .\end{cases}
$$

\section{Green's Function}

Let $\mathbf{f}(\cdot)=\left(\begin{array}{l}f_{1}(\cdot) \\ f_{2}(\cdot)\end{array}\right)$ be a continuous vector-valued function. Now, we derive Green's function of problem (1)-(7). Consider the inhomogeneous eigenvalue problem consisting of the differential system,

$$
\begin{aligned}
& u_{2}^{\prime}(x)-\left(p_{1}(x)+\lambda\right) u_{1}(x)=f_{1}(x), \\
& u_{1}^{\prime}(x)+\left(p_{2}(x)+\lambda\right) u_{2}(x)=-f_{2}(x), \quad x \in I,
\end{aligned}
$$

and boundary conditions (2), (3) and transmission conditions (4)-(7) with $\lambda$ are not an eigenvalue of problem (1)-(7).

Now we can represent the general solution of (56) in the following form:

$$
\mathbf{u}(x, \lambda)=\left\{\begin{aligned}
A_{1}\left(\begin{array}{l}
\phi_{11}(x, \lambda) \\
\phi_{21}(x, \lambda)
\end{array}\right) & \\
+B_{1}\left(\begin{array}{l}
\chi_{11}(x, \lambda) \\
\chi_{21}(x, \lambda)
\end{array}\right), & x \in\left[a, c_{1}\right), \\
A_{2}\left(\begin{array}{l}
\phi_{12}(x, \lambda) \\
\phi_{22}(x, \lambda)
\end{array}\right) & \\
+B_{2}\left(\begin{array}{l}
\chi_{12}(x, \lambda) \\
\chi_{22}(x, \lambda)
\end{array}\right), & x \in\left(c_{1}, c_{2}\right), \\
A_{3}\left(\begin{array}{l}
\phi_{13}(x, \lambda) \\
\phi_{23}(x, \lambda)
\end{array}\right) & \\
+B_{3}\left(\begin{array}{l}
\chi_{13}(x, \lambda) \\
\chi_{23}(x, \lambda)
\end{array}\right), & x \in\left(c_{2}, b\right] .
\end{aligned}\right.
$$

We applied the standard method of variation of the constants to (57); thus, the functions $A_{i}(x, \lambda)$ and $B_{i}(x, \lambda)(i=$ $1,2,3)$ satisfy the linear system of equations

$$
\begin{aligned}
& A_{1}^{\prime}(x, \lambda) \phi_{21}(x, \lambda)+B_{1}^{\prime}(x, \lambda) \chi_{21}(x, \lambda)=f_{1}(x), \\
& A_{1}^{\prime}(x, \lambda) \phi_{11}(x, \lambda)+B_{1}^{\prime}(x, \lambda) \chi_{11}(x, \lambda)=-f_{2}(x), \\
& x \in\left[a, c_{1}\right), \\
& A_{2}^{\prime}(x, \lambda) \phi_{22}(x, \lambda)+B_{2}^{\prime}(x, \lambda) \chi_{22}(x, \lambda)=f_{1}(x), \\
& A_{2}^{\prime}(x, \lambda) \phi_{12}(x, \lambda)+B_{2}^{\prime}(x, \lambda) \chi_{12}(x, \lambda)=-f_{2}(x), \\
& x \in\left(c_{1}, c_{2}\right), \\
& A_{3}^{\prime}(x, \lambda) \phi_{23}(x, \lambda)+B_{3}^{\prime}(x, \lambda) \chi_{23}(x, \lambda)=f_{1}(x), \\
& A_{3}^{\prime}(x, \lambda) \phi_{13}(x, \lambda)+B_{3}^{\prime}(x, \lambda) \chi_{13}(x, \lambda)=-f_{2}(x), \\
& x \in\left(c_{2}, b\right] .
\end{aligned}
$$

Since $\lambda$ is not an eigenvalue and $\omega_{i}(\lambda) \neq 0(i=1,2,3)$, each of the linear systems in (58) has a unique solution which leads to

$$
\begin{array}{r}
A_{1}(x, \lambda)=\frac{1}{\omega(\lambda)} \int_{x}^{c_{1}} \chi^{\top}(\xi, \lambda) \mathbf{f}(\xi) d \xi+A_{1}, \\
B_{1}(x, \lambda)=\frac{1}{\omega(\lambda)} \int_{a}^{x} \phi^{\top}(\xi, \lambda) \mathbf{f}(\xi) d \xi+B_{1}, \\
x \in\left[a, c_{1}\right), \\
A_{2}(x, \lambda)=\frac{\delta^{2}}{\omega(\lambda)} \int_{x}^{c_{2}} \chi^{\top}(\xi, \lambda) \mathbf{f}(\xi) d \xi+A_{2}, \\
B_{2}(x, \lambda)=\frac{\delta^{2}}{\omega(\lambda)} \int_{c_{1}}^{x} \phi^{\top}(\xi, \lambda) \mathbf{f}(\xi) d \xi+B_{2}, \\
A_{3}(x, \lambda)=\frac{\gamma^{2}}{\omega(\lambda)} \int_{x}^{b} \chi^{\top}(\xi, \lambda) \mathbf{f}(\xi) d \xi+A_{3},
\end{array}
$$




$$
\begin{array}{r}
B_{3}(x, \lambda)=\frac{\gamma^{2}}{\omega(\lambda)} \int_{\mathcal{C}_{2}}^{x} \phi^{\top}(\xi, \lambda) \mathbf{f}(\xi) d \xi+B_{3}, \\
x \in\left(c_{2}, b\right],
\end{array}
$$

where $A_{i}, B_{i}(i=1,2,3)$ are arbitrary constants and

$$
\begin{aligned}
\phi(\xi, \lambda)= \begin{cases}\left(\begin{array}{l}
\phi_{11}(\xi, \lambda) \\
\phi_{21}(\xi, \lambda)
\end{array}\right), & \xi \in\left[a, c_{1}\right), \\
\left(\begin{array}{l}
\phi_{12}(\xi, \lambda) \\
\phi_{22}(\xi, \lambda)
\end{array}\right), & \xi \in\left(c_{1}, c_{2}\right), \\
\left(\begin{array}{l}
\phi_{13}(\xi, \lambda) \\
\phi_{23}(\xi, \lambda)
\end{array}\right), & \xi \in\left(c_{2}, b\right],\end{cases} \\
\chi(\xi, \lambda)= \begin{cases}\left(\begin{array}{l}
\chi_{11}(\xi, \lambda) \\
\chi_{21}(\xi, \lambda)
\end{array}\right), & \xi \in\left[a, c_{1}\right), \\
\left(\begin{array}{l}
\chi_{12}(\xi, \lambda) \\
\chi_{22}(\xi, \lambda)
\end{array}\right), & \xi \in\left(c_{1}, c_{2}\right), \\
\left(\begin{array}{l}
\chi_{13}(\xi, \lambda) \\
\chi_{23}(\xi, \lambda)
\end{array}\right), & \xi \in\left(c_{2}, b\right] .\end{cases}
\end{aligned}
$$

Substituting (59) into (57), we obtain the solutions of (56),

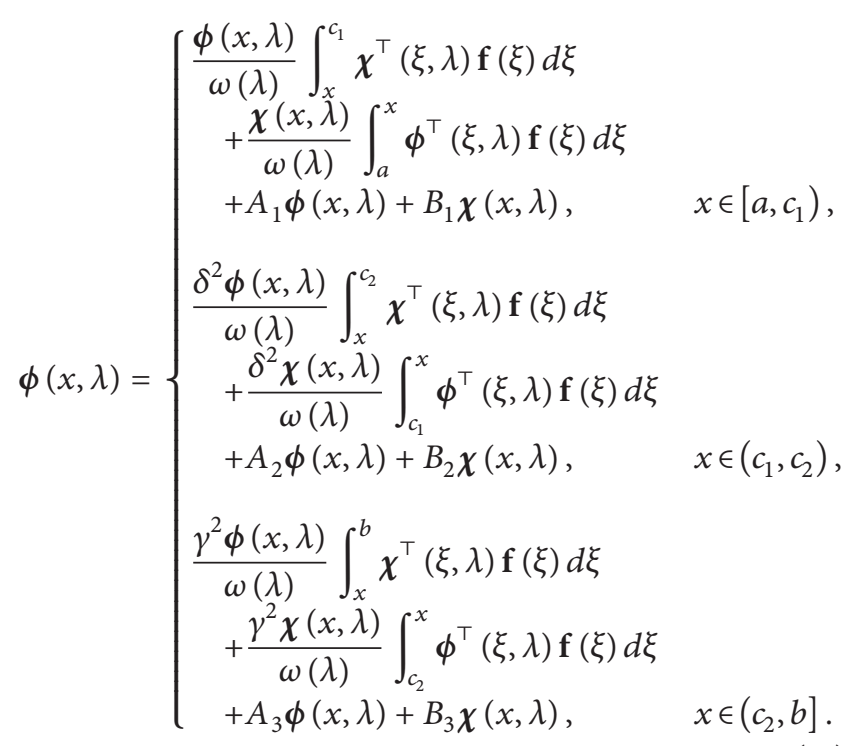

Then, from boundary conditions (2), (3) and transmission conditions (4)-(7), we get

$$
\begin{aligned}
A_{1}= & \frac{\gamma^{2}}{\omega(\lambda)} \int_{\mathcal{C}_{2}}^{b} \chi^{\top}(\xi, \lambda) \mathbf{f}(\xi) d \xi \\
& -\frac{\delta^{2}}{\omega(\lambda)} \int_{\mathcal{C}_{1}}^{\mathcal{C}_{2}} \chi^{\top}(\xi, \lambda) \mathbf{f}(\xi) d \xi
\end{aligned}
$$

$$
\begin{aligned}
B_{1}= & 0 \\
A_{2}= & \frac{\gamma^{2}}{\omega(\lambda)} \int_{\mathcal{C}_{2}}^{b} \chi^{\top}(\xi, \lambda) \mathbf{f}(\xi) d \xi \\
B_{2}= & -\frac{1}{\omega(\lambda)} \int_{a}^{c_{1}} \phi^{\top}(\xi, \lambda) \mathbf{f}(\xi) d \xi \\
A_{3}= & 0, \\
B_{3}= & \frac{\delta^{2}}{\omega(\lambda)} \int_{c_{1}}^{c_{2}} \phi^{\top}(\xi, \lambda) \mathbf{f}(\xi) d \xi \\
& -\frac{1}{\omega(\lambda)} \int_{a}^{c_{1}} \phi^{\top}(\xi, \lambda) \mathbf{f}(\xi) d \xi .
\end{aligned}
$$

Then (61) can be written as

$$
\begin{aligned}
\mathbf{u}(x, \lambda)= & \int_{a}^{c_{1}} \mathbf{G}(x, \xi, \lambda) \mathbf{f}(\xi) d \xi \\
& +\delta^{2} \int_{c_{1}}^{c_{2}} \mathbf{G}(x, \xi, \lambda) \mathbf{f}(\xi) d \xi \\
& +\gamma^{2} \int_{\mathcal{C}_{2}}^{b} \mathbf{G}(x, \xi, \lambda) \mathbf{f}(\xi) d \xi,
\end{aligned}
$$

where

$$
\mathbf{G}(x, \xi, \lambda)= \begin{cases}\frac{\chi(x, \lambda) \phi^{\top}(\xi, \lambda)}{\omega(\lambda)}, & a \leq \xi \leq x \leq b, \\ \frac{\chi^{\top}(\xi, \lambda) \phi(x, \lambda)}{\omega(\lambda)}, & a \leq x \leq \xi \leq b, \\ & x \neq c_{1}, c_{2}, \xi \neq c_{1}, c_{2},\end{cases}
$$

The function $\mathrm{G}(x, \xi, \lambda)$ is called Green's function of problem (1)-(7). Obviously, $\mathbf{G}(x, \xi, \lambda)$ is a meromorphic function of $\lambda$, for every $(x, \xi) \in I^{2}$, which has poles only at the eigenvalues.

\section{Conflict of Interests}

The authors declare that there is no conflict of interests regarding the publication of this paper.

\section{Disclosure}

This paper is in final form and no version of it will be submitted for publication elsewhere.

\section{References}

[1] A. N. Tikhonov and A. A. Samarskiŭ, Equations of Mathematical Physics, Pergamon Press, Oxford, UK, 1990.

[2] A. V. Likov and A. Yu. Mikhailov, The Theory of Heat and Mass Transfer, Gosnergoizdat, Moscow, Russia, 1963. 
[3] B. Chanane, "Eigenvalues of Sturm-Liouville problems with discontinuity conditions inside a finite interval," Applied Mathematics and Computation, vol. 188, no. 2, pp. 1725-1732, 2007.

[4] M. Kadakal and O. Sh. Mukhtarov, "Sturm-Liouville problems with discontinuities at two points," Computers \& Mathematics with Applications, vol. 54, no. 11-12, pp. 1367-1379, 2007.

[5] N. Altinisik, M. Kadakal, and O. Sh. Mukhtarov, "Eigenvalues and eigenfunctions of discontinuous Sturm-Liouville problems with eigenparameter-dependent boundary conditions," Acta Mathematica Hungarica, vol. 102, no. 1-2, pp. 159-175, 2004.

[6] B. M. Levitan and I. S. Sargsjan, Sturm-Liouville and Dirac Operators, vol. 59, Kluwer Academic, Dordrecht, The Netherlands, 1991.

[7] N. B. Kerimov, "A boundary value problem for the Dirac system with a spectral parameter in the boundary conditions," Differential Equations, vol. 38, no. 2, pp. 164-174, 2002.

[8] M. H. Annaby and M. M. Tharwat, "On the computation of the eigenvalues of Dirac systems," Calcolo, vol. 49, no. 4, pp. 221240, 2012.

[9] M. H. Annaby and M. M. Tharwat, "The Hermite interpolation approach for computing eigenvalues of Dirac systems," Mathematical and Computer Modelling, vol. 57, no. 9-10, pp. 24592472, 2013.

[10] B. Keskin and A. S. Ozkan, "Inverse spectral problems for Dirac operator with eigenvalue dependent boundary and jump conditions," Acta Mathematica Hungarica, vol. 130, no. 4, pp. 309-320, 2011.

[11] M. M. Tharwat, A. Yildirim, and A. H. Bhrawy, "Sampling of discontinuous Dirac systems," Numerical Functional Analysis and Optimization, vol. 34, no. 3, pp. 323-348, 2013.

[12] M. M. Tharwat, "On sampling theories and discontinuous Dirac systems with eigenparameter in the boundary conditions," Boundary Value Problems, vol. 65, 2013.

[13] R. Kh. Amirov, "On system of Dirac differential with discontinuity conditions inside an interval," Ukrainian Mathematical Journal, vol. 57, pp. 712-727, 2005.

[14] B. M. Levitan and I. S. Sargsjan, Introduction to Spectral Theory: Selfadjoint Ordinary Differential Operators, Translation of Mathematical Monographs, vol. 39, American Mathematical Society, Providence, RI, USA, 1975.

[15] V. F. Zhdanovich, "Formulae for the zeros of Dirichlet polynomials and quasi-polynomials," Doklady Akademii Nauk SSSR, vol. 135, no. 8, pp. 1046-1049, 1960. 


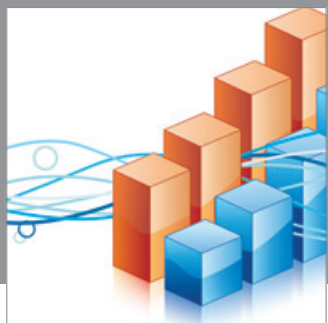

Advances in

Operations Research

mansans

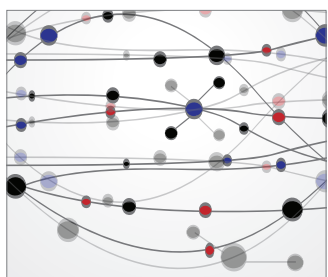

The Scientific World Journal
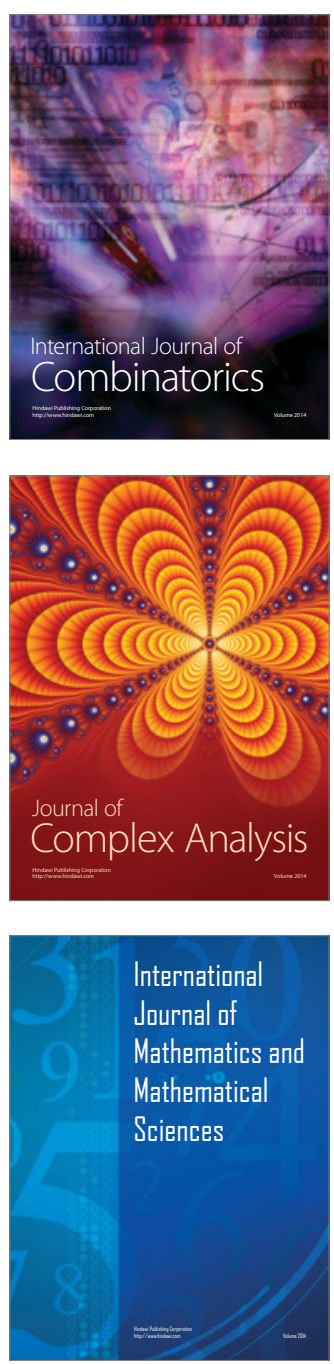
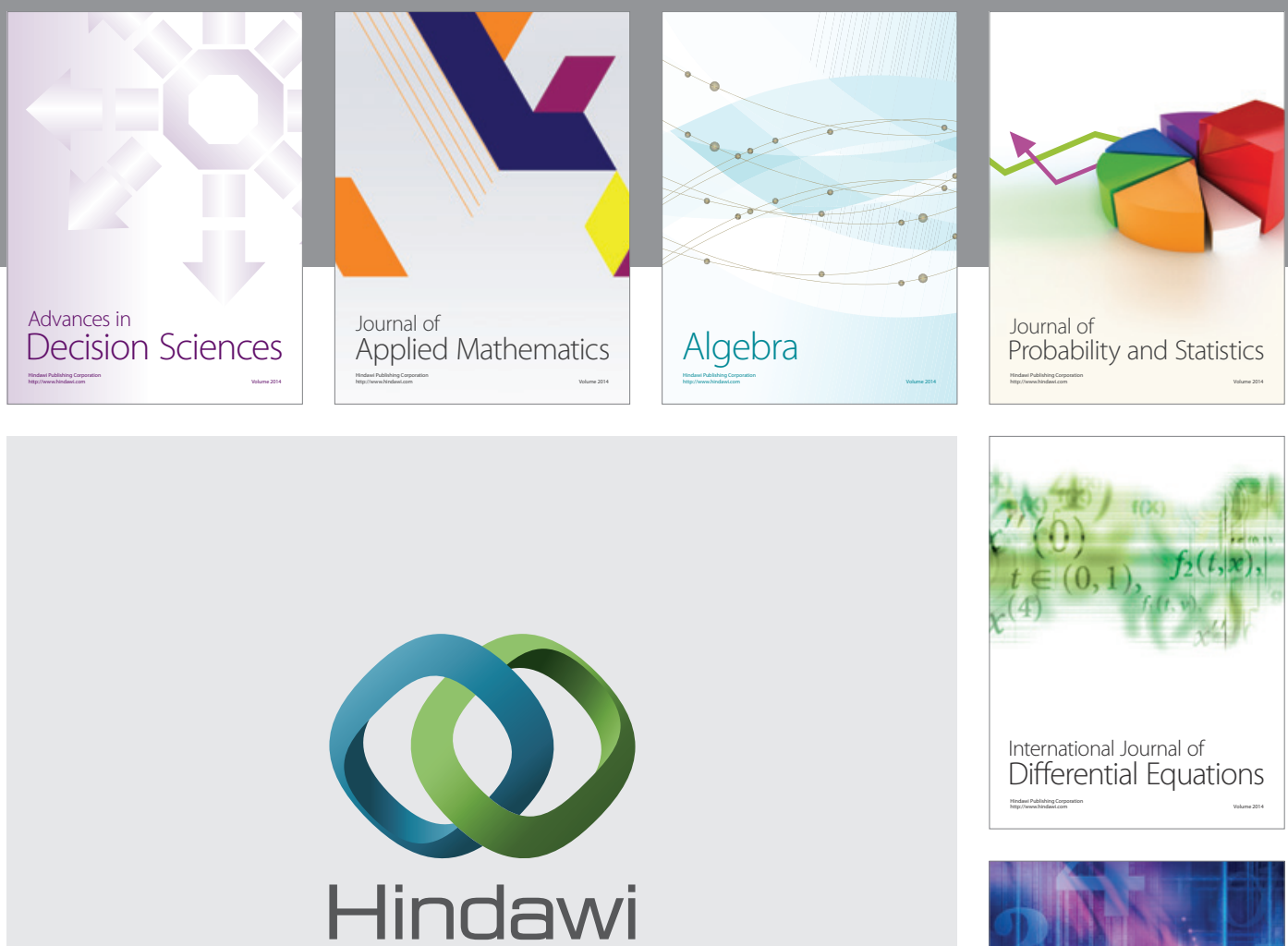

Submit your manuscripts at http://www.hindawi.com
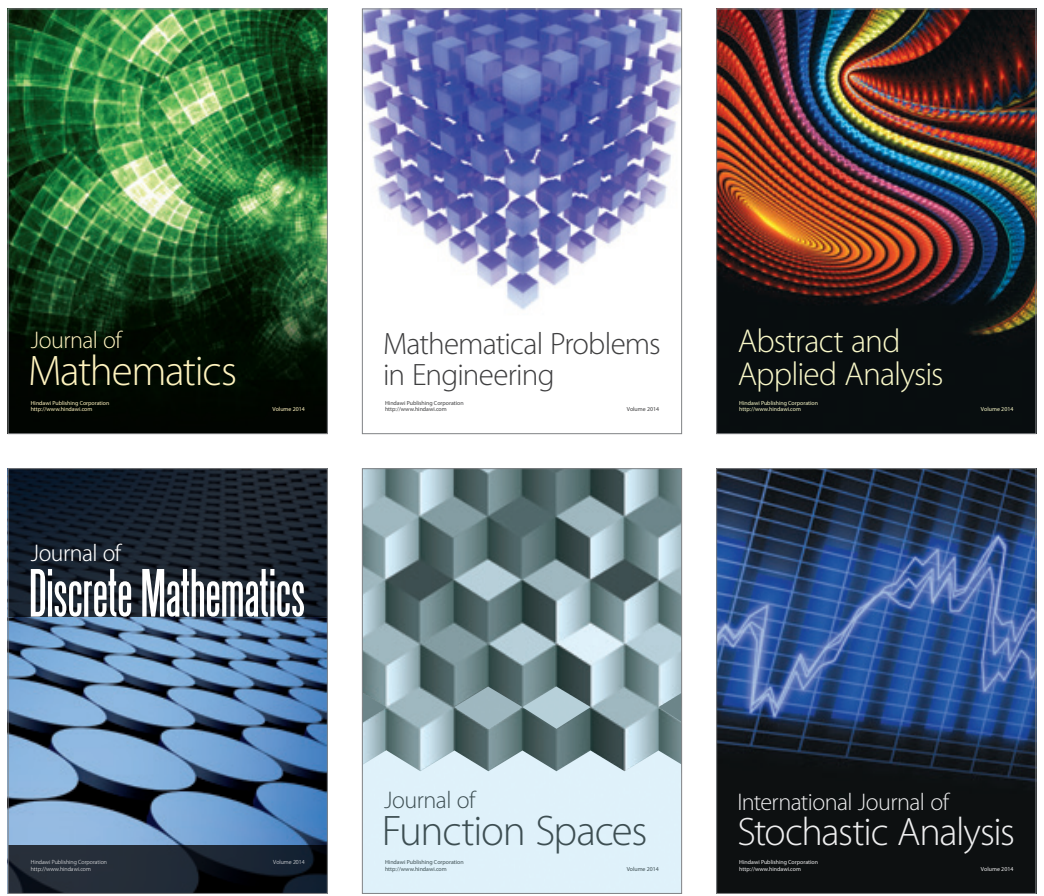

Journal of

Function Spaces

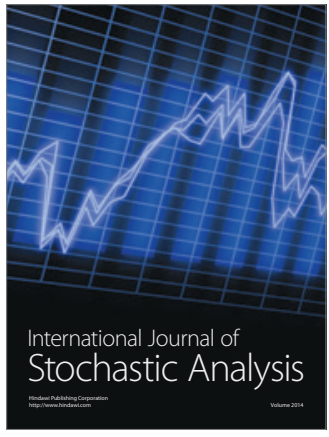

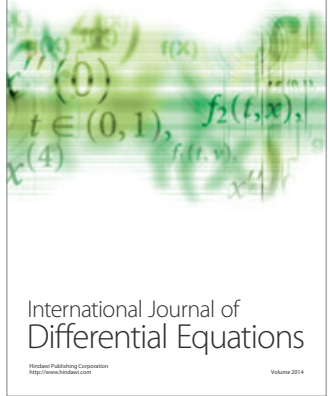
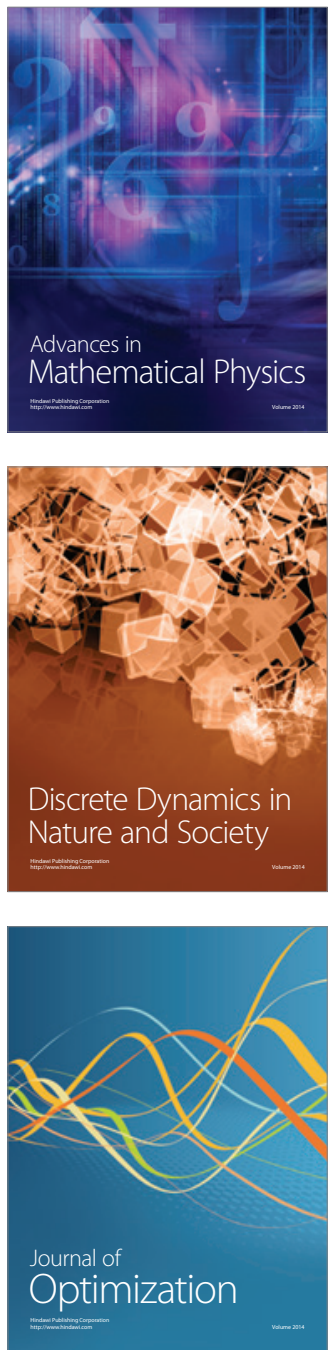